Vittalle - Revista de Ciências da Saúde v. 31, n. 1 (2019) 52-60
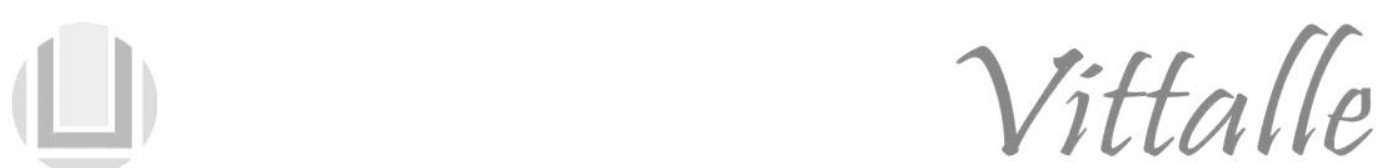

\title{
Cuidados de enfermagem ao paciente oncológico portador de cateter totalmente implantado
}

\author{
Diego Augusto Lopes Oliveira ${ }^{a,}$, Rodrigo de Almeida Fontes ${ }^{b}$, Maria Betânia da Silva ${ }^{b}$ \\ ${ }^{\mathrm{a}}$ Centro Universitário Tabosa de Almeida (ASCES-UNITA), Caruaru, PE, Brasil \\ ${ }^{\mathrm{b}}$ Centro Universitário Estácio do Recife, Recife, PE, Brasil
}

Histórico do Artigo
Recebido em:
25/01/2019
Aceito em:
16/04/2019

Palavras-chave:
Cateteres de demora;
Cuidados de
Enfermagem;
Enfermagem
Oncológica;
Enfermagem.

Keywords:

Catheters Indwelling;

Nursing Care;

Oncology Nursing;

Nursing.

\begin{abstract}
RESUMO
Os avanços tecnológicos propiciam muitos benefícios na terapia endovenosa com o uso do cateter venoso central totalmente implantado, estes exigem dos profissionais de enfermagem uma apropriação de conhecimento e capacitação especifica para a sua utilização envolvendo diversos saberes para oferta de um cuidado centrado nas necessidades do paciente. Nesse sentido objetivou-se, com este estudo, identificar os cuidados de enfermagem, prestados pelo enfermeiro, com o cateter venoso central totalmente implantado, em pacientes oncológicos. Trata-se de um estudo descritivo, transversal com abordagem quantitativa. A coleta dos dados utilizou checklist elaborado pelos autores e foi aplicado junto a vinte enfermeiros em um Centro de Oncologia de um hospital universitário da cidade do Recife-PE nos meses de setembro e outubro de 2017. O estudo foi aprovado pelo comitê de ética na pesquisa da referida instituição. Para análise dos dados utilizou-se estatística descritiva e análise fatorial. O perfil da amostra era do sexo feminino (100\%), na faixa etária de 25 a 36 anos (40\%), com mais de 10 anos de formação $(75 \%)$ e especialização na área de oncologia $(60 \%)$. Nas respostas do checklist observou-se que as profissionais conheciam os aspectos relacionados a prevenção de infecção, tipo de agulha para punção e cuidados após punção do dispositivo. Os profissionais não utilizam o processo de enfermagem como metodologia orientadora. Entende-se que os profissionais apresentam conhecimento satisfatório sobre os cuidados com o dispositivo necessitando de treinamento/orientação especificas para normatização e oferta de maior segurança e qualidade em seu cuidado.
\end{abstract}

Nursing care to oncological patient with fully implanted catheter

\section{ABSTRACT}

The technological advances provide many benefits in intravenous therapy with the use of the fully implanted central venous catheter, these require from nursing professionals an appropriation of knowledge and specific qualification for their use involving several knowledge to offer care centered on the patient's needs. In this sense, the objective of this study was to identify the nursing care provided by the nurse, with the central venous catheter fully implanted in oncologic patients. This is a descriptive, cross-sectional study with a quantitative approach. Data collection used a checklist elaborated by the authors and was applied to twenty nurses in an Oncology Center of a university hospital in the city of Recife-PE in September and October 2017. The study was approved by the Ethics Committee in the research of the aforementioned institution. Descriptive statistics and factorial analysis were used for data analysis. The profile of the sample was female $(100 \%)$, in the age group from 25 to 36 years $(40 \%)$, with more than 10 years of training $(75 \%)$ and specialization in the field of oncology $(60 \%)$. In the answers of the checklist it was observed that the professionals knew the aspects related to infection prevention, type of needle for puncture and care after puncture of the device. The professionals do not use the nursing process as a guiding methodology. It is Understood that professionals present satisfactory knowledge about the care of the device requiring specific training/guidance for standardization and offering greater safety and quality in their care.

\footnotetext{
*Autor correspondente: diegoaugusto.enf@gmail.com (Oliveira D.A.L)
} 


\section{Introdução}

O avanço tecnológico na área médica e, em especial, na terapia via endovenosa, propiciou o uso de materiais como o cateter totalmente implantado. Este exige dos profissionais de enfermagem a apropriação de conhecimento específico para sua utilização. $O$ conceito de tecnologia na enfermagem vai além do uso de materiais e equipamentos, já que envolve diversos saberes que conduzem à finalidade proposta pelo processo produtivo do profissional enfermeiro que é o cuidado integral (1).

A preocupação progressiva com o nível de qualidade no cuidado tem se tornado objeto principal de muitos sistemas de saúde em todo o mundo, com o intuito de garantir assistência satisfatória ao paciente. No entanto, mesmo com os avanços tecnológicos que permitiram a criação de novos tratamentos, aparelhos e protocolos assistenciais, os pacientes continuam expostos a diversos riscos quando submetidos aos cuidados em saúde, particularmente, em ambientes hospitalares (2).

A utilização de cateteres venosos tem se destacado em pacientes oncológicos por se tratar de um acesso vascular seguro quando comparado com outros tipos de dispositivos. A possibilidade do acesso venoso central possibilita inúmeras ações terapêuticas e intervencionistas que promoveram o progresso da saúde e da qualidade de vida ao longo das últimas décadas (3).

$\mathrm{Na}$ área oncológica, o Cateter Venoso Central Totalmente Implantado (CVC-TI) é uma opção para os pacientes adultos, pois garante segurança na administração de medicamentos endovenosos a longo prazo. Este cateter está indicado para aqueles que serão submetidos à quimioterapia de longa duração (superior a seis meses), oferecendo, aos pacientes, redução da dor e da ansiedade ocasionadas por repetidas punções ou dissecções venosas para receber a terapia indicada, além de maior liberdade e segurança para desenvolver suas atividades diárias, o que contribui para uma melhor qualidade de vida (4).

O CVC-TI vem sendo utilizado desde 1983, tornando-se essencial, pois possibilita a infusão de quimioterápicos, hemocomponentes e nutrição parenteral, além de coleta de sangue (3). A manipulação do CVC-TI não é caracterizada como atividade privativa do enfermeiro. A enfermagem é de grande importância para os pacientes que fazem uso do cateter venoso central totalmente implantado, tendo em vista que ele é o profissional da equipe multidisciplinar habilitado para manusear todo sistema de CVC-TI, realizando curativos, punções e outros procedimentos (5).

A ausência de consensos técnicos relacionados ao desenvolvimento de ações de enfermagem voltadas para atenção específica ao portador de CVC-TI, a ausência de evidências científicas que instrumentem um processo de educação permanente respaldado prejudicam o desenvolvimento desta ação junto aos pacientes, comprometendo a continuidade do tratamento e sua segurança. Tratar uma ação especializada de forma generalista impede a criação de estratégias de melhoria contínua e mitigação dos riscos associados à assistência. Este estudo teve como objetivo avaliar o conhecimento de enfermeiros para desenvolvimento de cuidados de enfermagem com CVC-TI em pacientes oncológicos.

\section{Materiais e métodos}

Estudo transversal, descritivo com abordagem quantitativa realizado em um hospital universitário da cidade do Recife-PE, Brasil, no qual é desenvolvida atenção integral ao paciente oncológico. O cenário da coleta dos dados foi o Centro de Oncologia Adulto e pediátrico no qual aplicou-se instrumento de coleta de dados com vinte profissionais enfermeiros selecionados de forma intencional, sendo incluídos os que trabalharam no 
serviço com tempo de atuação superior a um ano, por compreender construção da habilidade específica e vivência do procedimento de forma a estabelecer rotina para o cuidado. Foram excluídos profissionais que apresentavam vínculo relacionado a liderança no serviço pesquisado.

$\mathrm{O}$ instrumento utilizado foi elaborado pelos autores, baseado em revisão da literatura voltada à temática (5). O mesmo foi disposto em formato de checklist com quatorze perguntas, cada uma com apenas uma alternativa correta e dividido em duas partes: a primeira relacionados a dados que possibilitassem a caracterização do participante a partir da coleta de dados sociodemográficos e a segunda com questões relacionadas a assistência de enfermagem ao paciente portador de CVC-TI. A coleta ocorreu nos meses de setembro e outubro de 2017 mediante assinatura do termo de consentimento livre e esclarecido pelos profissionais, em local reservado e adequado a resposta do instrumento não oferecendo condições que causassem transtornos a atividade laborativa dos envolvidos. O estudo teve aprovação pelo comitê de ética em pesquisa (CEP) do Hospital Universitário Osvaldo Cruz - Universidade de Pernambuco (HUOC/UPE) através do CAAE $\mathrm{n}^{\circ}$ 77541617.0.0000.5640.Os dados coletados foram tabulados utilizando o programa Microsoft Excel® 2014 e analisados através de estatística descritiva simples e análise fatorial.

\section{Resultados}

A tabela 1 apresenta o perfil da amostra no tocante a coleta dos dados sociodemográficos e profissionais. Há a predominância de profissionais do sexo feminino (100\%), com ano de formação acadêmica entre 2001 e 2010 (45\%), especialização em sua maioria em áreas divergentes a oncologia (65\%), desenvolvendo atividades assistenciais em regime plantonistas (75\%). Estas profissionais têm tempo de atuação na atenção ao paciente oncológico em tempo inferior a 5 anos (60\%) possuindo, em sua maioria, 02 vínculos empregatícios profissionais (75\%).

Observa-se que as frequências evidenciadas nas respostas relacionadas a não especialização na área de oncologia (25\%) e no tempo de atividade no setor de oncologia >11anos (20\%) apresentam relevância no conteúdo das respostas do instrumento de coleta de dados quando relacionadas as perguntas específicas da assistência de enfermagem ao portador de CVC-TI.

Tabela 1 -Perfil sociodemográfico e profissional dos enfermeiros atuantes no Centro de Oncologia. Recife, PE, 2017.

\begin{tabular}{cr}
\hline VARIÁVEL & N $(\%)$ \\
\hline GẾNERO & \\
Masculino & $0(0)$ \\
Feminino & $20(100)$ \\
FAIXA ETÁRIA & \\
$25-36$ & $9(45)$ \\
$37-48$ & $8(40)$ \\
$49-52$ & $2(10)$ \\
Não respondeu & $1(5)$ \\
ANO DE FORMAÇÃO & \\
$1990-2000$ & $5(25)$ \\
$2001-2010$ & $9(45)$ \\
$>2011$ & $6(30)$ \\
\hline
\end{tabular}




\begin{tabular}{cr}
\hline VARIÁVEL & N $(\%)$ \\
\hline INSTITUIÇÃO DE ENSINO SUPERIOR (IES) & \\
Pública & $15(75)$ \\
Privada & $5(25)$ \\
PÓS-GRADUAÇÃo OU ESPECIALIZAÇÃO/ÁREA & \\
Oncologia & $5(25)$ \\
Especialização em outra área & $13(65)$ \\
Sem especialização & $2(10)$ \\
FUNÇÃo & \\
Diarista & $5(25)$ \\
Plantonista & $15(75)$ \\
TEMPO DE ATUAÇÃ̃O NETOR DE ONCOLOGIA & $12(60)$ \\
C5 anos & 41 anos \\
$>11$ anos & $4(20)$ \\
NÚMEROS DE VINCULOS EMPREGATÍCIOS & \\
01 vínculo & $05(25)$ \\
02 vínculos & $15(75)$ \\
\hline TOTAL & $20(100)$ \\
\hline
\end{tabular}

A tabela 2 evidencia a frequência das respostas obtidas nas questões específicas relacionadas a assistência prestada pelo enfermeiro ao paciente portador de CVC-TI. As questões remetiam os cuidados para prevenção e controle de infecção do cateter, tipo de agulha utilizada na punção, cuidados pós punção, especificamente quanto ao curativo, solução antisséptica para pele e cuidados para manutenção e desobstrução. Outro aspecto abordado durante a coleta foi da utilização da Sistematização da Assistência de Enfermagem (SAE) como metodologia orientadora do cuidado prestado.

Tabela 2 - Frequência das respostas relacionadas aos cuidados de enfermagem ao paciente portador de CVC-TI, Recife, PE, 2017.

\begin{tabular}{lr}
\multicolumn{1}{c}{ VARIÁVEL } & N (\%) \\
\hline Cuidados para Prevenção de Infecção no CVC-TI & $17(85)$ \\
Antissepsia do local de punção e aplicação de curativo estéril & $02(10)$ \\
Aplicação de curativo oclusivo estéril e SF 0,9\% & $01(5)$ \\
Não respondeu & \\
Tipo de agulha na Punção do Reservatório do CVC-TI & $19(95)$ \\
Hubber e Cytocan® & $01(5)$ \\
Agulha 25x0,7 e Cytocan® & \\
Como realizar o Curativo após punção & $11(55)$ \\
Clorexidina® Alcoólica e Tegaderm® ${ }^{\circledR}$ & $03(15)$ \\
Fixar a Agulha com gaze e Esparadrapo ou Filme Transparente Semipermeável & $05(25)$ \\
Outra resposta & $01(5)$ \\
Não respondeu & \\
Tipos de Solução para Antissepsia no local do Cateter & $10(50)$ \\
Clorexidina alcoólica & $03(15)$ \\
Clorexidinadegermante & $\mathbf{0 7}(\mathbf{3 5})$ \\
\hline Outras respostas & \\
\hline
\end{tabular}




\begin{tabular}{lc}
\hline \multicolumn{1}{c}{ VARIÁVEL } & N (\%) \\
\hline Solução para desobstrução do CVC - TI & $07(35)$ \\
Heparina & $04(20)$ \\
Soro Fisiológico à 0,9\% & $08(40)$ \\
Outros & $01(05)$ \\
Não respondeu & \\
Ângulos de introdução da agulha para punção do Reservatório do CVC - TI & $18(90)$ \\
$90^{\circ}$ & $02(10)$ \\
$45^{\circ}$ & \\
Sinais flogísticos (Procedimentos adotados) & $14(70)$ \\
Comunicar a Equipe Médica/Realizar Cultura & $05(25)$ \\
Outros & $01(5)$ \\
Não respondeu & \\
Implantação do SAE para pacientes com CVC - TI & $09(45)$ \\
Sim & $11(55)$ \\
Não & $20(100)$ \\
\hline TOTAL & \\
\hline
\end{tabular}

A tabela 2 ainda evidencia que as respostas dos enfermeiros relacionadas a assistência específica do enfermeiro ao portador de CVC-TI apresentam-se satisfatórias no tocante aos cuidados relacionados a prevenção de infecção (85\%), tipo de solução e técnica para antissepsia (50\%), cuidados após punção do cateter (55\%), dispositivos adequados para realização da punção (95\%)e cuidados relacionados a presença de sinais flogísticos evidenciados durante a avaliação do paciente $(70 \%)$. Os itens relacionados a ângulo de realização da punção, solução utilizada para desobstrução do cateter e utilização da SAE para realização da prática apresentaram-se como insatisfatórios na amostra estudada.

\section{Discussão}

O perfil dos profissionais de enfermagem entrevistados é predominantemente feminino, podendo ser explicado, em parte, devido às construções culturais que vinculam o cuidado a responsabilidade feminina desde os primórdios, justificando a predominância principalmente pela figura feminina nesta profissão (6). Em estudo nacional realizado pelo Conselho Federal de Enfermagem (COFEN) observou-se que a profissão é uma categoria é predominantemente jovem $(61,7 \%$ dos profissionais tem até 40 anos) e hegemonicamente feminina (85\%), a equipe de enfermagem brasileira é considerada majoritariamente não-branca (quando somados pretos e pardos, com percentuais de $11,5 \%$ e $41,5 \%$, respectivamente) $(7,8)$.

Quando comparado ao perfil da amostra deste estudo evidencia-se semelhança na predominância de sexo e faixas etárias esta se dá por estas características serem fortes no setor saúde, tendo esta área maioria da força de trabalho composta por mulheres em idade jovem, representando atualmente mais de $70 \%$ de todo o contingente, sendo esperado o crescimento dessa tendência nos próximos anos, embora, no caso da enfermagem, os dados também demonstrem uma presença crescente do contingente masculino $(9,10)$.

A assistência de enfermagem desenvolvida ao paciente portador de CVC-TI tem corpo de conhecimento específico na especialidade de enfermagem oncológica, dado que não é confirmado na amostra da pesquisa. A realização do procedimento não é uma 
atividade privativa do enfermeiro oncologista, porém entende-se que este apresenta corpo de conhecimento e habilidades específicas para tal atividade. Sabe-se que o índice de enfermeiros especialistas em oncologia ainda é muito baixo e se constitui um dado preocupante tendo em vista que se faz necessário a locação de outros profissionais não especializados na área, causando assim uma preocupação em capacitar esses profissionais para estarem habilitados a prestar a assistência eficaz aos pacientes de forma integral.

Cabe aos profissionais de saúde, em especial aos de enfermagem, um melhor conhecimento e domínio sobre estes cateteres, sua manipulação, respeitando a assepsia do mesmo, de modo a minimizar as complicações infecciosas, tendo em conta que grande parte dos portadores tem baixa imunidade, uma vez que estão sob tratamento quimioterápico (11). Estas medidas impedem invasão de microrganismos através do túnel do cateter resultando em infecção e até em sepse. É necessário que o enfermeiro, ao puncionar o CVC-TI, tenha consciência que a sua função durante o procedimento não é apenas garantir a infusão de medicamentos por aquela via, mas garantir também que a técnica aplicada é segura (12).

A infecção é a mais frequente complicação relacionada ao uso de cateter central, podendo levar à remoção do dispositivo precocemente, ocorrendo tanto na loja subcutânea, na qual o port está instalado, quanto ao longo do túnel subcutâneo onde o cateter está inserido, colocando o paciente em risco de sepse. A melhor maneira de preveni-la é por meio da utilização de técnica estéril durante o manuseio do cateter; além da obediência ao prazo estabelecido para a troca da agulha, equipo e conexões, de 48h $(13,14)$. Dentre os procedimentos fortemente recomendados pelo Center for Disease Control and Prevention (CDCP), a higienização das mãos e a desinfecção das linhas de infusão, são medidas para a prevenção de infecção relacionada deste tipo de cateter venoso central (15).

Para que o dispositivo tenha maior durabilidade e que se evite o aparecimento desta complicação, é necessário que os enfermeiros que lidam com o mesmo possuam conhecimento técnico-científico e capacitação para seu manuseio, de forma articulada e padronizada, uma vez que esta é uma atividade que deve ser desenvolvida exclusivamente por enfermeiros (16).

O preparo da pele é um passo importante da técnica de punção e para tal, o antisséptico de escolha, é a clorexidina alcoólica, que é mais efetiva, uma vez que a ação microbicida mantém efeito residual na pele por tempo superior a outras substâncias com a mesma finalidade. É importante destacar que a antissepsia da pele deve ser realizada por meio de movimentos em espiral, de dentro para fora, a partir do centro do port e que devem ser executados por, pelo menos, três vezes antes da inserção da agulha. A primeira conduta, em caso de suspeita de infecção do CVC-TI, é realizar hemocultura de via periférica e da via central, a fim de identificar o sítio de infecção e o microrganismo envolvido, orientando a escolha do antibiótico a ser utilizado ou a retirada do cateter (4). Estes cuidados devem ser estendidos a todas as medidas de contato desde a manipulação, punção e curativos após a administração dos fármacos. Torna-se essencial a observância e orientação dos profissionais para estas medidas.

$\mathrm{O}$ acesso ao dispositivo deve ser feito por meio de punção do reservatório, com agulha não cortante (tipo Hubber), introduzida num ângulo de noventa graus até tocar o fundo do reservatório, e o principal cuidado inclui o flush com solução fisiológica e heparinização (4). Em relação à agulha, a recomendação é que se use apenas a não cortante ou tipo Huber para a punção do port, pois o uso da agulha hipodérmica pode fissurar o septo de silicone (15). A utilização da agulha em angulação divergente da recomendada pode culminar em erros de procedimento pela não acessibilidade da 
câmara e não confirmação do correto posicionamento da mesma, visto que para técnica recomendada este passo é importante para execução. Nos casos de ausência do retorno venoso, resistência à infusão e/ou dor à infusão, o profissional deve interrompê-la e avaliar o posicionamento adequado da agulha (4).

No que se refere a coberturas/curativo, a inserção do cateter deve ser protegida com cobertura, gaze, filme transparente ou semipermeável estéril; trocar o curativo com gaze estéril a cada 48 horas ou antes, se sujo, solto ou úmido, e a cada 7 dias para curativos com transparente semipermeável ou antes, se sujo, solto ou úmido; em pacientes com discrasias sanguíneas, sangramento local, sudorese excessiva, optar por curativos com gaze estéril; durante o banho proteger com plástico (16).Sempre que este cateter estiver em uso (infusão de medicamentos ou fluídos) é necessário que no local de inserção da agulha se faça um curativo que tem como objetivo estabilizar a agulha (17).

Dentre as complicações relacionadasa manipulação do CVC-TI observa-se a obstrução da agulha de punção, normalmente relacionada a da formação de trombos, fibrina ou precipitação de drogas. A principal conduta para prevenir os casos de obstrução de cateter é a lavagem com solução salina, regularmente, entre a administração de dois ou mais medicamentos e após o uso do dispositivo, seguida da heparinização, cuja solução deve ter concentração final de $100 \mathrm{UI} / \mathrm{ml}$ (18).

A SAE é regulamentada no Brasil como um método que organiza o trabalho profissional, organizado em cinco etapas inter-relacionadas: coleta de dados, diagnóstico de enfermagem, planejamento, implementação e avaliação de enfermagem (19). Seu desenvolvimento é indispensável e tem por finalidade identificar os problemas reais ou potenciais do cliente, de forma a subsidiar o plano de cuidados e atender as necessidades encontradas prevenindo as complicações (20).

Acredita-se que a utilização de instrumentos que padronizem o cuidado pode reforçar o desejo natural dos profissionais enfermeiros em melhorar a assistência, ao mesmo tempo em que representa a melhor forma de realizar os procedimentos específicos, oferecendo subsídios para direcionar os enfermeiros a observar, documentar e avaliar os pacientes portadores de CVC-TI, bem como acompanhar os cuidados a eles inerentes e, desse modo, poder adaptá-los às características de cada serviço $(16,20)$.

Identificou-se como limitação a realização da aplicação de um instrumento em apenas um momento e a não observação do desenvolvimento da prática continuamente nem a avaliação dos pacientes posteriormente aos procedimentos.

\section{Conclusão}

Constatou-se que o conhecimento dos profissionais de enfermagem avaliados se apresentou satisfatório para as ações de prevenção de infecção (tipo de solução e antissepsia), cuidados na punção do cateter, dispositivos adequados para punção e cuidados relacionados a rotina de avaliação quanto a sinais de complicação neste dispositivo. Evidenciou-se como insatisfatórias as ações relacionadas a angulação de realização da punção que não se apresentou conforme em sua totalidade, demonstrando lacunas no processo de trabalho e educação permanente da instituição, solução utilizada para desobstrução do cateter e utilização da SAE para desenvolvimento do cuidado.

Entende-se que há a necessidade de desenvolvimento de medidas que fortaleçam o campo de conhecimento de enfermagem relacionado a esta prática, sobretudo por ser observado na literatura informações divergentes sobre o desenvolvimento desta prática. Medidas como padronização de procedimentos através de ferramentas de gestão e qualificação como os Procedimentos Operacionais Padrão (POP), algoritmos e mapas de orientação podem otimizar a conformidade e segurança nas ações destes profissionais. 
Recomenda-se a realização de estudos voltados ao entendimento das lacunas de conhecimento e formação específica para os profissionais de enfermagem, haja vista ter sido observado, nos resultados deste estudo, maior concentração de enfermeiros não especialistas no desenvolvimento de atenção especializada.

Desenvolver medidas relacionadas ao treinamento e desenvolvimento de pessoal, observação e controle dos pacientes no tocante as possíveis complicações do CVC-TI oriundas da assistência de enfermagem e implementar a SAE emergem como medidas importantes para fortalecer o campo de conhecimento e garantir maior respaldo e segurança na atenção a este grupo de pacientes.

\section{Referências}

1. Pinto AH, Lange C, Muniz RM, Azevedo NA, Genz N, Almeida NLD. Cateter totalmente implantado e o conhecimento da equipe de enfermagem oncológica. Rev Enferm UFPE on line2015; 9(11):9663-70.

2. Alves KYA, Costa TD, Barros AG, Lima KYN, Santos VEP. Segurança do paciente na terapia intravenosa em unidade de terapia intensiva. Rev Fundam Care 2016;8(1):3714-3724.

3. Pacheco GC, Beserra GES, Oselame GB, Neves EB. Conhecimento do Enfermeiro em Relação ao Cateter Totalmente Implantado. Revista Mineira de Enfermagem2014; 16 (2): 181-184.

4. Pires NN, Vasques CI. Conhecimento de enfermeiros acerca do manuseio de cateter totalmente implantado. Texto Contexto Enferm2014; 23(2): 443-50.

5. Vieira NN. Validação de manual de condutas para manuseio de cateter totalmente implantado. Dissertação [Mestrado em Enfermagem]. Brasilia: Universidade de Brasília; 2015.

6. Jesus APGAS, Yoshida NCP, Freitas JGAP. Prevalência da automedicação entre acadêmicos de Farmácia, Medicina, Enfermagem e Odontologia. Revista Estudos 2013; 40 (2):151-164.

7. Sala de Enfermagem [homepage da internet]. O perfil da enfermagem brasileira e sua relação com a população. Disponível em: https://saladeenfermagem.wordpress.com/2017/02/28/perfil-enfermageme-a-populacao/.

8. Machado MH, Filho WA, Lacerda WF, Oliveira E, Lemos W, WermelingerM, et al. Características gerais da enfermagem: o perfil sócio demográfico. Enferm Foco 2015; 6 (1/4): 11-17.

9. Ribeiro AC, Ramos LHD, Mandu ENT. Perfil sociodemográfico e profissional de enfermeiros de um hospital público de Cuiabá - MT. Ciênc Cuid Saúde 2014; 13 (4): 625-633.

10. Lima EFA, Borges JV, Oliveira ERA, Velten APC, Primo CC, Leite FMC. Qualidade de vida no trabalho de enfermeiros de um hospital universitário. Ver Eletr Enf 2013; 15 (4): 1000-06.

11. Santos SF, Viana RS, Alcoforado CLGC, Campos CC, Matos SS, Ercole FF. Ações de enfermagem na prevenção de infecções relacionadas ao cateter venoso central: uma revisão integrativa. Revista SOBECC 2014; 19(4): 219-225.

12. Oliveira TF, Rodrigues MSC. Enfermagem na prevenção de infecção em cateter totalmente implantado no paciente oncológico. Cogitare Enferm 2016; 21 (2): 01-05.

13. Neves Junior MA, Melo RC, Goes Junior AMO, Protta TR, Almeida CC, Fernandes AR, et al. Infecções em cateteres venosos centrais de longa permanência: revisão da literatura. J Vasc Bras2010; 9 (1): 46-50.

14. Souza RAP, Carmo TG. Risco de infecção para o cliente oncológico em uso de cateter venoso central totalmente implantado - Revisão integrativa. R. Epidemiol Control Infec2017; 7(4): 01-11.

15. Correa ACP, Corrêa ACP, Araújo EF, Ribeiro AC, Pedrosa ICF.Perfil sociodemográfico e profissional dos enfermeiros da atenção básica à saúde de Cuiabá. Rev Eletr Enf 2012; 14 (1): 171-80.

16. Costa CAB. Bundle de cateter venoso central: conhecimento e comportamento dos profissionais de saúde da unidade de terapia intensiva adulto de um hospital de grande porte. Dissertação [Mestrado em Enfermagem]. Belo Horizonte:Universidade Federal de Minas Gerais; 2017.

17. Oliveira FT, Stipp MAC, Silva LD, Frederico M, Duarte SCM. Comportamento da equipe multiprofissional frente ao Bundle do Cateter Venoso Central na Terapia Intensiva. Escola Anna Nery 2016; 20 (1): 55-62.

18. Silva JP, Garanhani ML, Peres AM. Sistematização da Assistência de Enfermagem na graduação: um olhar sob o Pensamento Complexo. Rev. Latino-Am. Enfermagem 2015; 23 (1): 59-66. 
19. Alfaro-Lefreve R. Aplicação do processo de enfermagem: fundamentos para o raciocínio clínico. $8^{\mathrm{a}}$ ed. Porto Alegre: Artes Médicas; 2014.

20. Honório RPP, Caetano JÁ, Almeida PCA. Validação de procedimentos operacionais padrão no cuidado de enfermagem de pacientes com cateter totalmente implantado. RevBrasEnferm, 2011; 64 (5): 882-9. 